\title{
CHARACTERIZATION OF TH PROFILE THROUGH DETECTION OF SERUM CYTOKINE LEVELS IN PATIENTS WITH TUBERCULOSIS ASSOCIATED OR NOT WITH HIV INFECTION
}

THESIS. S. M. U. R. Silva submitted this dissertation for her Masters in Tropical Diseases at Botucatu School of Medicine, São Paulo State University, UNESP, Botucatu, São Paulo, Brazil, 2004.

Advisor: Professor Jussara Marcondes Machado

ABSTRACT: Tuberculosis, a thousand-year-old scourge, still continues to afflict mankind: nowadays there are two billion infected human beings, of whom $10 \%$ will be taken ill; among these, 3.5\% to $6.0 \%$ will lose their lives. AIDS, a pandemic caused by the human immunodeficiency virus (HIV) and that has been scaring since the eighties, favors tuberculosis development, as it causes deep changes in immunity. On the other hand, Mycobacterium tuberculosis increases the viral load of HIVinfected patients, which results in a loss in life expectancy. In a co-infected person, tuberculosis has a faster clinical evolution, a more difficult diagnosis due to: the lack of characteristic chest radiographs, an often inconclusive cutaneous hypersensitivity response to purified protein derivative (PPD), and the low concentration of bacillus in the sputum; all of these are used to show tuberculosis activity. The overall result is a very fast evolution of both diseases during co-infection, which reduces the patient's life expectancy and makes necessary the search for markers that can suggest the existence of tuberculosis in HIV-infected patients. This study was aimed to assess Th profile through the demonstration of serum cytokine levels in patients with AIDS by the time tuberculosis was diagnosed as an opportunistic disease; so it could be used as an alternative predictive test together with blood T CD4+ lymphocyte count, allowing that co-infection be evidenced. For this purpose, three groups were formed: G1 (or control group), with 25 blood-donors; G2, with 20 HIV-negative patients with tuberculosis; and G3, with 22 HIV-positive patients co-infected with tuberculosis. In G3, viral load as well as T CD4+ and T CD8+ cell counting were also assessed. 
Blood samples for serum cytokines determination were obtained from all patients after tuberculosis diagnosis and before the start of anti-tuberculosis treatment; in G3, T CD4+ lymphocytes and viral load were assessed until 15 days after that. The results showed that serum levels of tumor necrosis factor-alfa (TNF- $\alpha$ ) in both groups of patients were significantly higher than those found in the control group; in G3, these levels were even higher than in G2. The levels of interferon-gamma (IFN- $\gamma$ ), which is also a Th1 profile cytokine, were significantly increased in G2 and G3, although $\mathrm{G} 2$ presented the highest level of all. No statistical difference was found between G1 and G2 interleukin-2 (IL-2) levels. Also, there were no statistical differences between the IL-2 serum levels in $\mathrm{G} 2$ and the control group; these groups showed higher IL-2 levels than G3. As to Th2 profile cytokines, a significant increase in interleukin-4 (IL-4) and interleukin-10 (IL-10) serum levels were found in both groups with active tuberculosis, and in $\mathrm{G} 3$, the levels were found to be even higher. Based on the serum levels of cytokines, it was possible to determine the Th profiles of patients in this study. This way, Th0 profile, defined as the concomitant of Th1 and Th2 cytokines, was found in all G2 patients with tuberculosis. Among the co-infected G3 patients, $50 \%$ of them showed Th0 profile and $50 \%$, Th2 profile. Nevertheless, Th0 profile of G3 patients differed from that of G2. In G3, serum levels of IL-2 and IFN- $Y$ were lower, and those of IL-4 and IL-10 were higher than in G2 (with tuberculosis only), indicating that, in $\mathrm{G} 3$, the Th0 profile was already turning into Th2. The author suggests that assessment of Th profile in patients with AIDS may be a useful marker to diagnose tuberculosis activity. This serum marker may be helpful to decide on the early introduction of anti-tuberculosis treatment, which can prevent $M$. tuberculosis from developing drug-resistance and save the patient's lives.

KEY WORDS: AIDS, tuberculosis, Mycobacterium tuberculosis, cytokines.

\section{CORRESPONDENCE TO:}

S. M. U. R. SILVA, Departamento de Doenças Tropicais e Diagnóstico por Imagem, Faculdade de Medicina de Botucatu, UNESP, Distrito de Rubião Junior, s/n, 18618000, Botucatu, SP, Brasil. Phone: 55143811 6212. Email: imachado@fmb.unesp.br 